\title{
FEBRILE-INFECTION-RELATED EPILEPSY SYNDROME (FIRES)
}

Researchers at Tel Aviv University, Israel, and other epilepsy centers in Taiwan; Rome, Italy; Boston, USA; Paris, France; Vogtatreuth and Kiel, Germany; and Salzburg, Austria studied retrospectively the pathogenesis, treatment, and outcome of 77 children diagnosed with febrile infection-related epilepsy syndrome (FIRES) and reported in 8 publications between 2001 and 2010. Median age at onset was 8 years (range 3-17 years), and the male-to-female ratio was 4:3. Prolonged refractory status epilepticus or multiple daily seizures (partial or secondarily generalized) were preceded by fever for a median of 4 days and nonspecific infection (upper respiratory or gastrointestinal) in $96 \%$ of patients. Mechanical ventilation was required for a median duration of 41 days. CSF showed minimal pleocytosis (57\%) and oligoclonal bands (4/12) but the etiology was undetermined. Tests for viral infection were negative. Genetic test for SCN1A mutation was negative in 2 patients examined. Treatment with antiepileptic drugs or steroids was ineffective. Treatments of possible benefit included IVIG (in 2 of 30 patients), ketogenic diet (in 1 of 4), and prolonged barbiturate anesthesia (1 of 46). Nine (12\%) patients died during the acute illness; of 68 survivors, $56(82 \%)$ had cognitive disabilities, and 63 (93\%) had refractory epilepsy at follow-up. Poor cognitive outcome was significantly associated with younger age at FIRES onset, and higher log of burst-suppression coma duration. Some patients had severe peripheral neuropathy and ataxia as sequelae. There was no correlation between mortality or degree of cognitive impairment and EEG foci (temporal or frontotemporal in 32 [54\%] of 59) during the acute phase, MRI abnormalities (signal hyperintensities and later atrophy in hippocampi), and duration of mechanical ventilation. (Kramer U, Chi C-S, Lin K-L, et al. Febrile infection-related epilepsy syndrome (FIRES): pathogenesis, treatment, and outcome. A multicenter study on 77 children. Epilepsia November 2011;52(11):1956-1965). (Respond: Uri Kramer MD, Pediatric Epilepsy Service, Tel Aviv Sourasky Medical Center 6 Weitzman St, Tel Aviv 64239, Israel. E-mail: umkramer@netvision.net.il).

COMMENT. Febrile infection-related epilepsy syndrome (FIRES) or "acute encephalitis with refractory, repetitive partial seizures" (AERRPS), as preferred in Japan, is a severe epileptic encephalopathy of undefined etiology and having a poor prognosis, with cognitive impairment, refractory epilepsy, and a high mortality rate.

One case-report, a febrile acute-onset epilepsy syndrome in a girl, resembling FIRES in some respects, tested positive for missense mutation of protocadherin 19 (PCDH19) gene located at Xq22. (Specchio N et al. Epilepsia Nov 2011;52(11):e172e175). The PCDH19 syndrome differs from FIRES in age at onset, ranging between 6 and 38 months and is restricted to females, whereas FIRES has an age at onset of 3-15 years and a male preponderance. The authors recommend testing for PCDH19 mutation in female acute-onset epilepsy resembling FIRES.

\section{ADVERSE EVENTS DURING INVASIVE EEG RECORDINGS}

Researchers in the Department of Paediatric Neurosurgery and Epilepsy Unit, Great Ormond Street Hospital, London, UK analyzed the hospital charts of 95 children operated on between 1995 and 2009 for medication-resistant focal epilepsy and requiring 